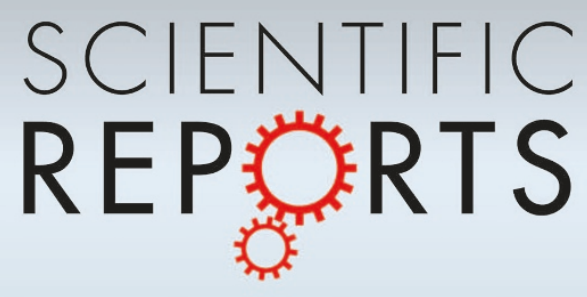

OPEN

SUBJECT AREAS:

CANCER PREVENTION

ADJUVANTS

PROTEIN VACCINES

ANTIGEN PRESENTATION

Received

8 January 2013

Accepted

22 May 2013

Published

6 June 2013

Correspondence and requests for materials should be addressed to S.D.M. (mengsd@im.

ac.cn)

* These authors contributed equally to this work.

\section{Placenta-derived gp96 as a multivalent prophylactic cancer vaccine}

\author{
Bao Zhao 1,2*, Yanzhong Wang ${ }^{1 *}$, Bo Wu', Shan Liu ${ }^{3}$, Erjie Wu' , HongXia Fan' ${ }^{1}$ MingMing Gui', \\ Lizhao Chen', Changfei Li' ', Ying Ju', Wei Zhang ${ }^{3}$ \& Songdong Meng ${ }^{1,2}$
}

${ }^{1}$ CAS Key Laboratory of Pathogenic Microbiology and Immunology, Institute of Microbiology, Chinese Academy of Sciences (CAS), Beijing, China, ${ }^{2}$ School of Life Sciences, University of Science and Technology of China, Hefei, China, ${ }^{3}$ Detection Center of Tumor Biology, Cancer Institute \& Hospital, Chinese Academy of Medical Sciences, Beijing, China.

A major challenge for designing prophylactic cancer vaccines is to define immunogenic and safe cancer antigens. Given the striking similarity of antigen expression patterns between cancer and embryonic tissues, we defined a prototype strategy of using placenta-derived heat shock protein gp96, which induces prophylactic anti-tumor $\mathrm{T}$ cell responses. Immunization with placental gp96 provided partial protection and long-term (at least 3 months) anti-tumor immunity against growth of transplantable melanoma or breast tumors in mice, elicited total protection against 7, 12-dimethylbenz(a)-anthracene (DMBA)-induced mammary tumors in rats, and significantly reduced the occurrence and growth of autochthonous breast tumors in HER2 transgenic mice. Placental gp96 activated HER2- and MUC1-specific T cell responses through binding to tumor-associated antigens. Our results reveal the novel immunogenicity of placental gp96 and its potential use as a multivalent cancer vaccine.

espite the remarkable progress in understanding the causes of cancer and the significant advances in cancer therapy in recent years, the disease persists, and the incidence of cancer is increasing worldwide ${ }^{1}$. Unlike the highly efficient prophylactic vaccines against infectious diseases, therapeutic vaccines against cancer that do not cause unacceptable autoimmune disorders have not been as effective, eliciting only incremental therapeutic effects ${ }^{2}$. The simplest explanation may be that using therapeutic vaccines to treat established tumors is equivalent to the unsuccessful approach of using hepatitis B virus (HBV) or human papilloma virus (HPV) vaccines to treat chronic HBV or HPV infection. Several count-back mechanisms have been reported for the involvement of tumor escape and immune suppression, likely driven by long-term tumor development, establishment, and growth, including impaired $\mathrm{T}$ cell responses, immune tolerance, and the suppressive tumor microenviroment, which likely act synergistically ${ }^{3}$. This underlines the need to develop a prophylactic vaccine approach for cancer that will provide low-cost and highly efficiency rationales.

A major challenge for designing prophylactic cancer vaccines is to define immunogenic and safe cancer antigens that can serve as targets for effective vaccines, including tumor-specific antigens and proteins overexpressed on the tumor but not on normal tissues. At present, only a limited number of cancer antigens have been found with few successes ${ }^{4-6}$. It is well documented that most solid tumor types express embryonic antigens to varying extents, and there is striking similarity of antigen expression between cancer and embryonic tissues, which provides the potential to target embryonic components as an effective strategy to prevent the appearance of cancers ${ }^{7}$. Indeed, vaccination with embryonic or stem cell antigens leads to a potent protective immune response against cancers ${ }^{8-10}$. As a temporary organ that perform nutrient and waste product exchanges between the mother and fetus, the placenta also displays higher cancer-associated gene expression, including IGF2, HIF-2 $\alpha$, GPC3, pregnancy-associated plasma protein A (PAPP-A), and MUC1 ${ }^{11}$.

As a member of the heat shock protein (HSP) 90 family, gp 96 has the unique ability to associate with antigenic peptides, presents these loaded antigens to both MHC class I and class II molecules, and activates specific T cells ${ }^{12,13}$. Our previous studies show that in hepatitis B virus (HBV)-infected liver cancer, gp96 binds virus-derived peptides and activates specific CTL responses by antigen presentation ${ }^{14,15}$. Moreover, recent studies provide compelling evidence of macrophages and dendritic cells activation by gp 96 through interaction with a subset of Toll-like receptors (TLRs) or CD91 ${ }^{16-19}$. Clinical trials using autologous gp96-peptide complexes as therapeutic vaccines have been initiated for treatment of a range of tumors with modest antitumor effects ${ }^{20}$. Based on the observations above, the aim of this study was to investigate whether placenta-derived gp96 (P-gp96) induces prophylactic anti-tumor $\mathrm{T}$ cell responses. 


\section{Results}

We first tested the ability of a placenta-derived gp96 vaccine to prevent tumors using tumor challenge assays. Gp96 protein was extracted from the placenta or liver tissues of C57BL/6 mice as previously described ${ }^{21}$. C57BL/6 mice were subcutaneously immunized three times with placenta-derived gp96 (P-gp96), gp96derived from liver (L-gp96), or PBS (no immunization) as a control. One week after the last immunization, mice were subcutaneously challenged with B16-F10 melanoma cells $\left(5 \times 10^{4}\right.$ cells/ mouse). Compared to L-gp96 or PBS, immunization with P-gp96 significantly inhibited tumor growth, decreasing tumor volume by approximately 49 or $45 \%$ at day 30 (both $P<0.01$ ) (Fig. 1a). Pgp96 immunization also dramatically enhanced the survival of tumor burdened mice through 50 days of observation (Fig. 1b). All PBS- or L-gp96-treated mice died within 40 days, whereas half of the P-gp96-treated mice survived at day 50. We further examined if P-gp96 could initiate a B16-specific T cell response. As seen in Fig. 1c, ELISPOT assay revealed that P-gp96 immunization resulted in $\sim 1$-fold increase of tumor-specific $\mathrm{T}$ cells compared to L-gp96 immunization. Additionally, as assessed by the killing assay using B16 cells as target cells, P-gp96 effectively elicited CTL with higher cytotoxicity than L-gp96 ( $P<0.01)$ (Fig. 1d).
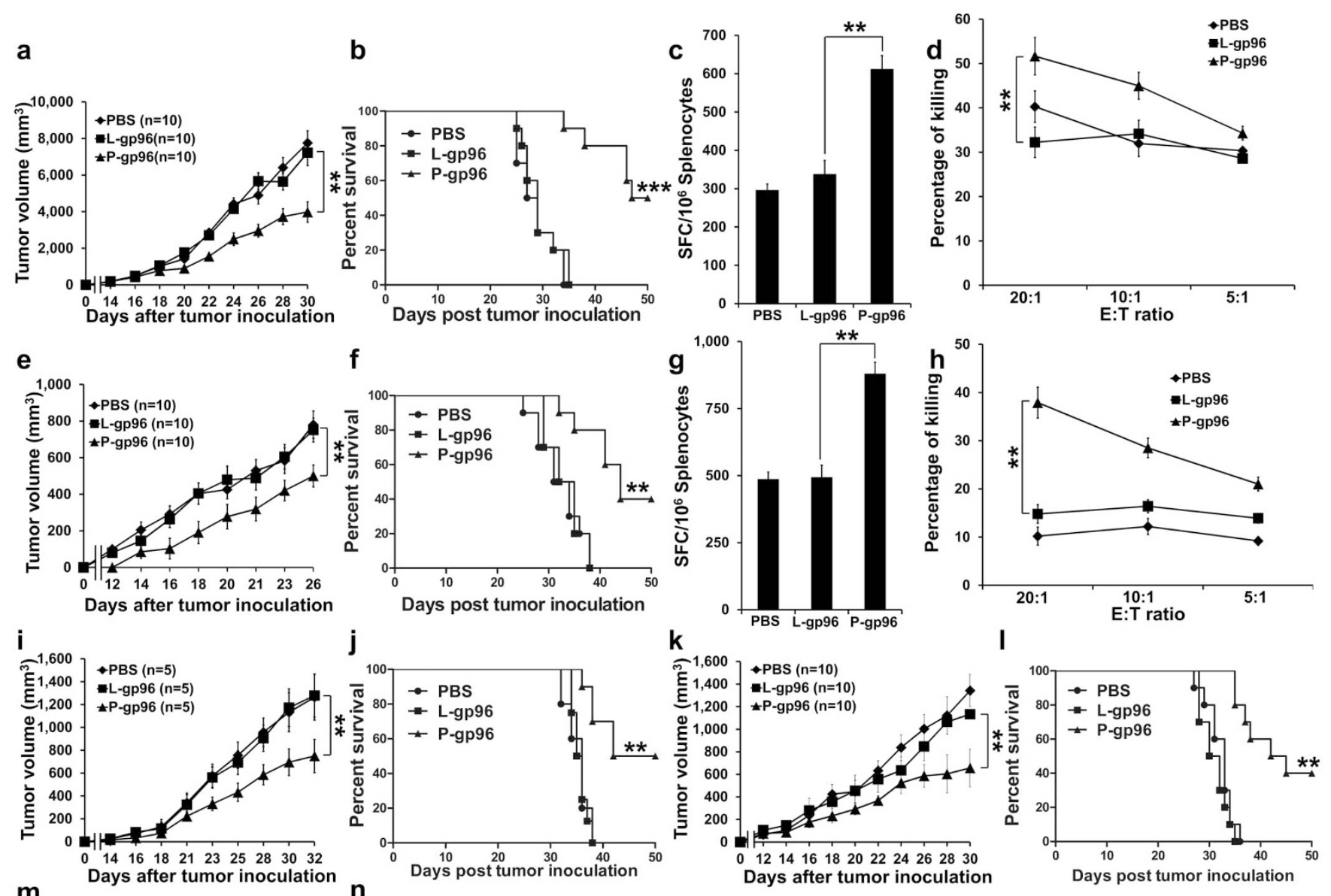

m

n
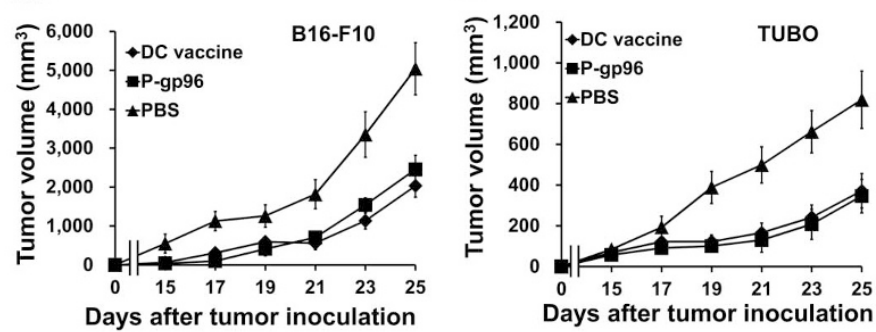

Figure $1 \mid$ Immunization with the P-gp96 vaccine induces antitumor T cell responses. Female C57BL/6 mice (a-d) or BALB/c mice (e-h) were immunized three times with P-gp96, L-gp96, or PBS. One week after the third immunization, the mice were subcutaneously challenged with $5 \times 10^{4}$ B16F10 cells or $6 \times 10^{5}$ TUBO cells. (a, e) Tumor burden was measured at 2-day intervals. (b, f) Kaplan-Meier plot of mouse survival. (c, g) Splenocytes from immunized mice were stimulated with B16-F10 (c) or TUBO (g) whole cell lysates antigens or BSA for background evaluation and assayed by IFN- $\gamma$ ELISPOT. (d, h) Splenocytes were stimulated with irradiated B16-F10 cells (d) or TUBO cells (h) in vitro for 3 days and analyzed for cytotoxic activity by FACS using CFSE-labeled B16-F10 cells or TUBO cells as target cells. (i, j) Growth of TUBO tumors (i) and mouse survival ( $j$ ) after adoptive transfer of $\mathrm{CD}^{+} \mathrm{T}$ cells from P-gp96-immunized mice into irradiated recipient BALB/c mice. ( $\left.\mathrm{k}, \mathrm{l}\right)$ TUBO tumor growth (k) and mouse survival (l) after P-gp96, L-gp96, or PBS immunization three times at 3-day intervals on the second day after tumor inoculation. Female C57BL/6 mice or BALB/c mice were immunized three times with DCs vaccine, $\mathrm{P}$-gp96, or PBS. One week after the third immunization, the mice were subcutaneously challenged with $5 \times 10^{4}$ B16-F10 cells or $6 \times 10^{5}$ TUBO cells. Growth of B16-F10 tumors (m) or TUBO tumors (n) was measured at 2-day intervals. Error bars indicate s.d. ** P $<$ $0.01,{ }^{* * *} \mathrm{P}<0.001$ (Analysis of variance (ANOVA) and post-hoc comparison using Tukey's test). 
We further examined the prophylactic efficiency of P-gp96 in a TUBO breast cancer mouse model. BALB/c mice were immunized with P-gp96 or L-gp96 derived from $\mathrm{BALB} / \mathrm{c}$ mice and challenged with TUBO breast cells $\left(6 \times 10^{5}\right.$ cells/mouse) one week after the last immunization. Similar results were observed in P-gp96-mediated tumor growth inhibition (Fig. 1e) and survival increase in tumorchallenged mice (Fig. 1f). Likewise, both the induction of TUBOspecific $\mathrm{T}$ cells (Fig. 1g) and the enhanced cytotoxicity of CTL (Fig. 1h) were detected in P-gp96-immunized mice compared to Lgp96-immunized mice. Moreover, adoptive transfer of $\mathrm{CD}^{+} \mathrm{T}$ cells from P-gp96-immunized but not L-gp96 immunized-mice resulted in significant inhibition of tumor growth (Fig. 1i) and enhanced survival (Fig. 1j) in mice challenged with TUBO cells. We also examined the therapeutic efficiency of P-gp96 in the TUBO breast cancer mouse model. P-gp96 immunization significantly inhibited TUBO tumor growth (Fig. 1k) and enhanced the survival (Fig. 11) of tumor burdened mice relative to L-gp96 or PBS immunization. In contrast to P-gp96, treatment with L-gp96 did not affect tumor growth and did not elicit a tumor-specific $\mathrm{T}$ cell response in either the B16 or TUBO model, which is consistent with previous studies ${ }^{19,22}$. To further evaluate the anti-tumor efficacy of P-gp96 vaccine, we compared the prophylactic efficiency of P-gp96 with a well-studied DC vaccine (DCs pulsed with tumor cell lysates) in B16 melanoma and TUBO breast cancer mouse models. As shown in Fig. $1 \mathrm{~m}$ and 1n, similar tumor inhibition effects were observed between P-gp96- and DCsimmunized mice.

Next, we tested whether P-gp96 immunization could provide long-term protective anti-tumor immunity. C57BL/6 mice were vaccinated three times with P-gp96 or L-gp96 derived from C57BL/6 mice. At three months after the last immunization, mice were subcutaneously inoculated with B16-F10 ( $5 \times 10^{4}$ cells/mouse). Compared to no treatment, tumor growth was slowed in mice treated with P-gp96 but not L-gp96 $(P<0.01)$ (Fig. 2a), and the survival of tumor burdened mice was also significantly enhanced $(P<0.001)$ (Fig. 2b). P-gp96-immunized mice exhibited a significant increase in B16-specific T cells by approximately 1.5 - or 2.5 -fold (Fig. 2c) and enhanced cytotoxicity of CTLs compared to L-gp96- or PBS-immunized mice (Fig. 2d). Similar results were observed in the TUBO model of P-gp96-mediated tumor growth inhibition (Fig. 2e), i.e., increased survival (Fig. 2f), a tumor-specific T cell response (Fig. 2g), and an increase in cytotoxicity (Fig. 2h). Together, these results indicate placenta-derived gp96 induced long-term $\mathrm{T}$ cell responses against transplanted tumors.

We also examined whether early immunization with P-gp96 isolated from rat placenta could prophylactically inhibit DMBAinduced mammary tumor development in rats. The body weight, tumor latency, tumor incidence, tumor volume, mean tumor weight, tumor multiplicity, and mammary histology data in P-gp96-immunized and -unimmunized rats are presented in supplementary Table S1. Whereas all unimmunized control rats developed breast tumors when the experiment was terminated at 21 weeks after DMBA treatment, none of the rats immunized with P-gp96 had any detectable mammary tumors (Fig. 3a), indicating that P-gp96 elicited total protection against DMBA-induced breast tumors. The median tumor volume of unimmunized rat was $6,262.63 \mathrm{~mm}^{3}$ compared to no tumors in P-gp96 immunized rats (Fig. 3b). Median number of mammary tumors per tumor-bearing rat (multiplicity) was also compared. The unimmunized rats had a range of 5 to 11 tumors per rat (median $=7.4)$ compared to none in P-gp96 immunized rats. Pathologic analyses revealed tumors classifications (supplementary Fig. S1).

In addition, $\mathrm{FVB} / \mathrm{N}-\mathrm{Tg}(\mathrm{MMTV}$ eu $) 202 \mathrm{Mul} / \mathrm{J}$ mice were used to further investigate the effect of P-gp96 immunization on growth of autochthonous breast tumors ${ }^{23}$. Mice were immunized three times with P-gp96 or L-gp96. Significant differences were observed in the time for tumor onset (median onset in P-gp96-immunized mice vs.

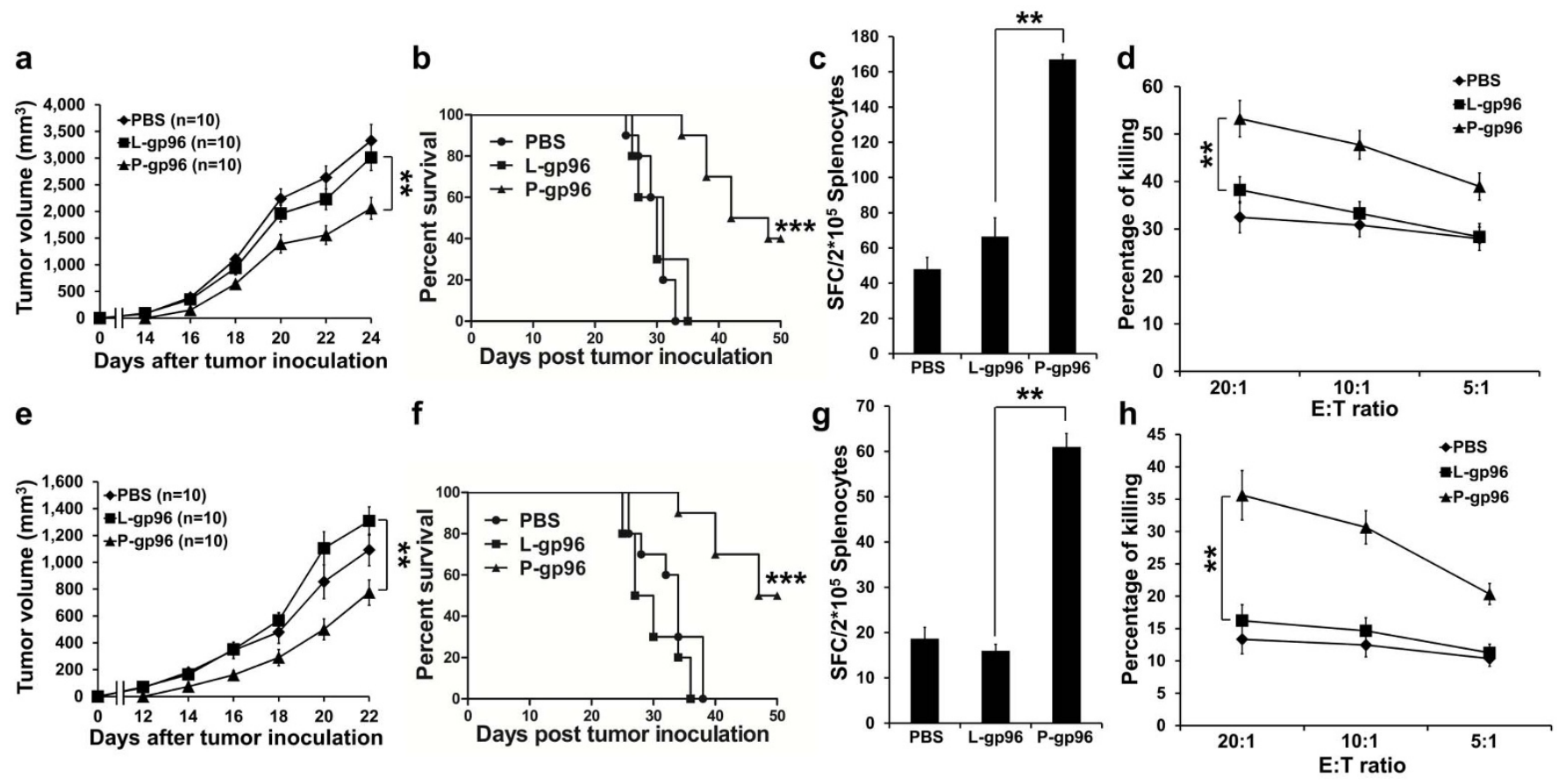

Figure $2 \mid$ P-gp96 vaccination induces long-term protective immunity against tumors. Female C57BL/6 (a-d) or BALB/c (e-h) mice were immunized three times with P-gp96, L-gp96, or PBS. Three months after the third immunization, the mice were subcutaneously challenged with $5 \times 10^{4}$ B16-F10 cells or $6 \times 10^{5}$ TUBO cells. (a, e) Tumor burden was measured at 2-day intervals. (b, f) Kaplan-Meier plot of mouse survival after the various vaccinations. (c, g) Splenocytes from immunized mice were stimulated with B16-F10 (c) or TUBO (g) whole cell lysates antigens or BSA for background evaluation and assayed by IFN- $\gamma$ ELISPOT. (d, h) Splenocytes were stimulated with irradiated B16-F10 (d) or TUBO (h) cells in vitro for 3 days and analyzed for cytotoxic activity by FACS using CFSE-labeled B16-F10 cells or TUBO cells as target cells. Error bars indicate s.d. ** P $<0.01$, $* * * \mathrm{P}<0.001$ (Analysis of variance (ANOVA) and post-hoc comparison using Tukey's test). 


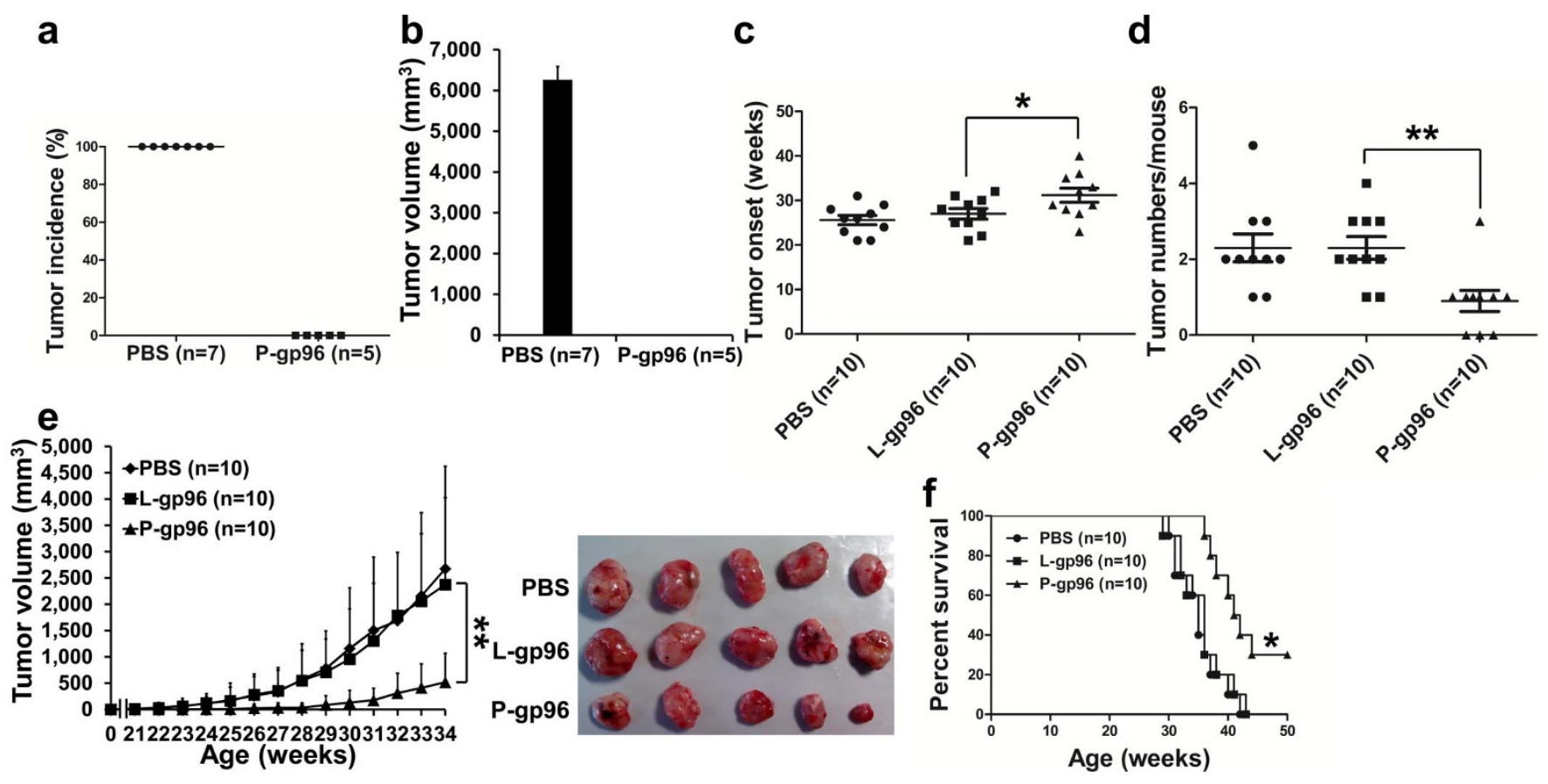

Figure 3 | P-gp96 immunization suppresses DMBA- or HER-2-induced mammary tumor development. (a, b) Tumor incidence (a) and growth of mammary tumors (b) in DMBA-treated rats immunized with P-gp96 or PBS. (c-f) P-gp96 immunization delays tumor onset in FVB/N$\mathrm{Tg}$ (MMTVneu) $202 \mathrm{Mul} / \mathrm{J}$ mice (c). Tumor number of palpable mammary carcinomas/mouse calculated at 34 weeks of age (d). Median tumor number was calculated as the cumulative number of tumors/total number of mice. Median tumor volumes in response to P-gp96 or L-gp96 immunization were measured at 1-week intervals (e, left), and representative images of subcutaneous tumors from PBS-, L-gp96 or P-gp96-treated mice at 34 weeks of age are shown (e, right). Mouse survival was calculated using the Kaplan-Meier method (f). Error bars indicate s.d. ${ }^{*} \mathrm{P}<0.05,{ }^{* *} \mathrm{P}<0.01$ (Analysis of variance (ANOVA) and post-hoc comparison using Tukey's test).

L-gp96-immunized or unimmunized mice, 31.2 vs. 27.0 or 25.6 weeks, $P<0.05$ ) (Fig. 3c), and the number of tumors developed per mouse up to 34 weeks (median tumor numbers in P-gp96-immunized mice vs. L-gp96-immunized or unimmunized mice, 0.9 vs. 2.3 or $2.3, P<0.01$ ) (Fig. $3 \mathrm{~d}$ ). Tumor growth was inhibited in mice treated with P-gp96 compared to L-gp96-immunized or unimmunized mice $(P<0.01)$ (Fig. 3e). In addition, immunization with the P-gp96 vaccine significantly enhanced the survival of tumor burdened mice (Fig. 3f).

Given that placenta-derived but not liver-derived gp96 exhibited high immunogenicity against multiple tumors, which relies heavily on the unique ability gp96 to associate with antigenic peptides within cells, we therefore assessed the expression of tumor-associated genes in the liver and placenta tissues. Analysis by quantitative real-time PCR showed dramatically higher expression of tumor-associated genes in the placenta than those in normal liver tissues of both mice and rats, including the extensively studied tumor antigens MUC1 and HER-2/ERBB2, and no significant differences were observed for housekeeping genes GAPDH and $\beta$-actin (Fig. 4a). Importantly, ELISPOT analysis of splenocytes from mice immunized with $\mathrm{P}$ gp96 showed that P-gp96 significantly increased a $\mathrm{K}^{\mathrm{d}}$-restricted HER-2 dominant epitope ${ }^{24}$ E63-specific T cell response (Fig. 4b). In contrast, L-gp96 or P-gp96 treated with trifluoroacetic acid (TFA) to release the associated peptides ${ }^{14}$ could not induce the E63-specific T cell response, indicating that gp96 in the placenta may bind HER-2-derived antigens. Furthermore, gp96-associated peptides from mouse placenta or liver were extracted with TFA and analyzed by matrix-assisted laser desorption/ionization (MALDI) mass spectrometry (Fig. 4c). Our results demonstrate that gp96 purified from the placenta binds to a large number of peptides ranging from 700 to $1,500 \mathrm{Da}$, whereas only a limited number of associated peptides were detected for L-gp96. These data suggest that gp96 binds tumor-associated antigens such as HER2, which is largely responsible for its immunogenicity against transplantable and autochthonous tumors that over-express (for instance) HER2 and MUC1.

Finally, we compared the expression levels of tumor-associated genes in human liver and placenta tissues. Analysis by quantitative real-time PCR showed dramatically higher expression of tumorassociated genes in the placenta relative to the liver, including the tumor antigens MUC1, HER2/ERBB2, MMP2, FGF2, c-Myc and VEGF-a (Fig. 4d). Human PBMCs were purified from the blood of HLA-A*0201 ${ }^{+}$healthy donors and incubated with P-gp96, TFAtreated P-gp96 (TFA-gp96), or PBS in vitro. IFN- $\gamma$ ELISPOT analysis using HLA-A*0201-restricted MUC1 or HER2 epitopes ${ }^{25}$ for stimulation revealed that $\mathrm{P}$-gp96 treatment remarkably expanded MUC1and HER2-specific CTLs compared to TFA-gp96 treatment or no treatment (PBS) (Fig. 4e). Meanwhile, P-gp96 treatment dramatically stimulated IFN- $\gamma$-producing T cells, but TFA-gp96 did not, as shown in ELISPOT assay with control peptide, indicating that Pgp96 may expand multiple peptide-specific $\mathrm{T}$ cell populations. Moreover, increased killing was observed when PBMCs were pulsed with P-gp96, using HLA-A2-positive breast cancer MCF-7 cells as target cell (Fig. 4f). These data suggest that the killing of this MUC1 over-expressing cell line by P-gp96-pulsed PBMC is likely to be due to activation of MUC1-specific T cells by P-gp96.

\section{Discussion}

Our study indicated that gp96 isolated from placenta initiates tumorspecific $\mathrm{T}$ cell responses via association with embryo-cancer antigens, and gp96 immunization provides prophylaxis against tumor xenografts, as well as carcinogen-induced or spontaneous cancers. Given the antigenic similarity between cancer and embryonic tissues ${ }^{7}$ and the capability of gp96 to bind the entire peptide repertoire generated within the cell ${ }^{13}$, our results therefore provide a rational basis for the development of a multivalent prophylactic cancer vaccine. 


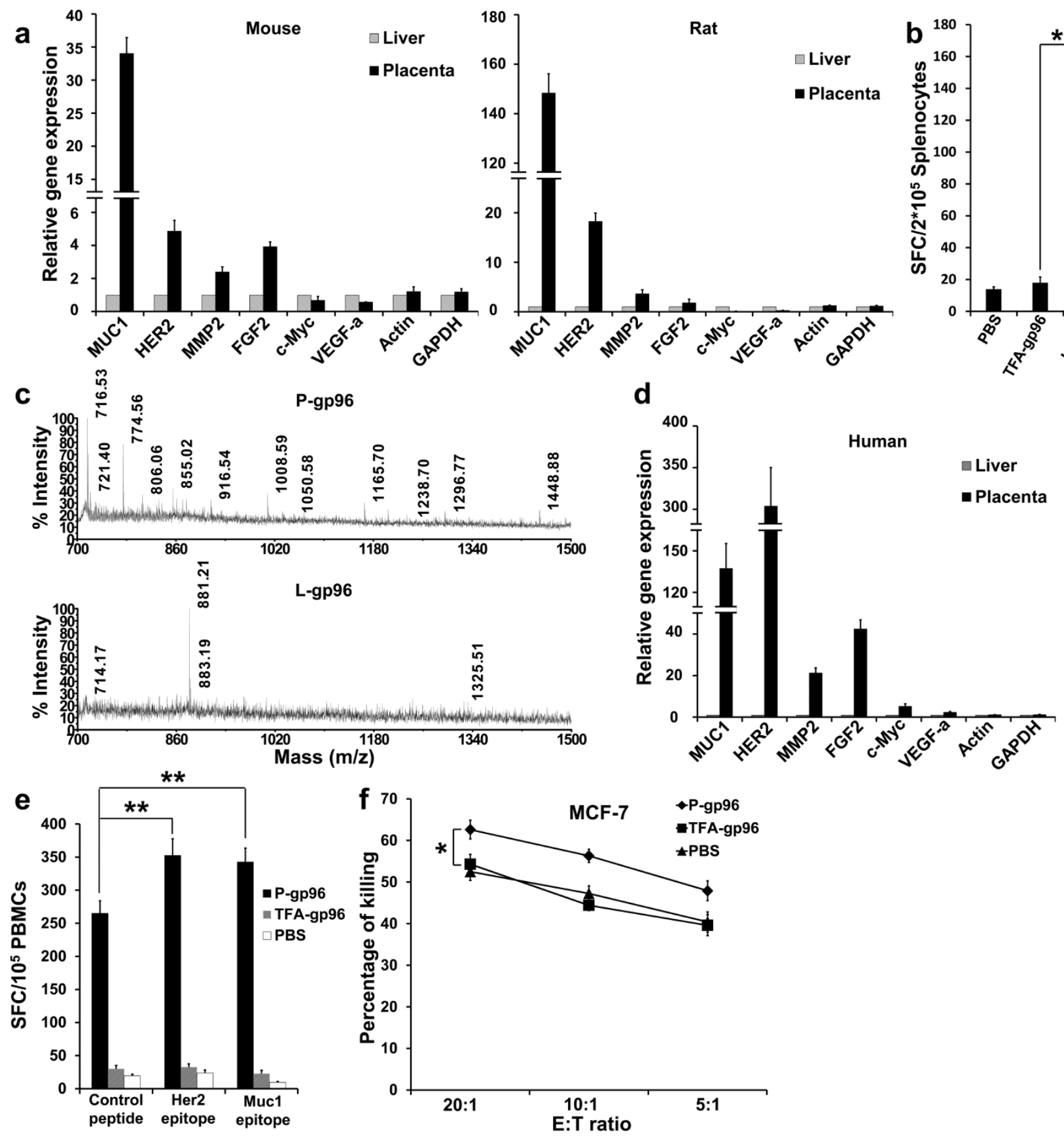

Figure $4 \mid$ Placenta-derived gp96 binds tumor-associated antigens. (a) Relative tumor associated-gene expression analysis of placenta and liver tissues in BALB/c mice $(n=5)$ or rats $(n=5)$ by real-time-PCR. The mRNA levels in liver tissues were arbitrarily set as 1.0. (b) Female BALB/c mice $(n=5)$ were immunized with P-gp96, L-gp96, TFA-gp96 or PBS as a control for three times. Splenocytes were isolated and stimulated with the mouse $\mathrm{K}^{\mathrm{d}}$-restricted

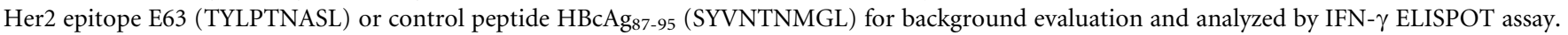
(c) Bulk peptides associated with P-gp96 or L-gp96 from BALB/c mice $(\mathrm{n}=3)$ were analyzed by MALDI mass spectrometry. Representative spectra are shown. (d) Relative tumor-associated gene expression between human placenta $(n=5)$ and liver tissue samples $(n=5)$ by real-time PCR analysis. (e, f) PBMCs from HLA-A*0201-positive healthy donors $(n=5)$ were stimulated with P-gp96, TFA-gp96, or BSA for 3 days. Then, stimulated PBMCs were either incubated with the HLA-A*0201-restricted Muc1 epitope (STAPPVHNV) or Her2 epitope (ILHNGAYSL) or a control peptide HBcAg $18-27$ (FLPSDFFPSV) for background evaluation and assayed by IFN- $\gamma$ ELISPOT (e) or analyzed for cytotoxic activity by FACS using CFSE-labeled MCF-7 cells as target cells (f). Error bars indicate s.d. ${ }^{*} \mathrm{P}<0.05$ and ${ }^{* *} \mathrm{P}<0.01$ (Two-tailed $t$-test or analysis of variance (ANOVA) and post-hoc comparison using Tukey's test).

There are several advantages of using P-gp96 as a prophylactic cancer vaccination strategy. First, clinical trials of autologous tumorisolated gp96 preparations have been initiated for treatment of multiple cancers, and no treatment-related severe adverse reactions or autoimmune diseases have been observed ${ }^{20}$. In addition, the potential autoimmune complications induced by P-gp96 may be avoided by targeting the vaccine to women in their post-child-bearing, premenopausal years at a higher risk of developing breast cancer. Second, unlike vaccination with embryonic stem cells, P-gp96 is readily available because the placenta is generally discarded after birth. Third, it is likely that P-gp96-mediated protection against cancer involves a number of shared embryo-cancer antigens, as evidenced by our 
observation that P-p96 activated specific T cells targeting HER2 and MUC1, which are highly expressed in the placenta and are the ideal targets for vaccination ${ }^{14}$. In addition, aside from its cross-presentation of antigenic peptides to MHC class I molecules, gp96 also interacts with TLRs and triggers the innate immunity that is essential for establishing anti-neoplastic immune responses ${ }^{17,18,26}$. Conceivably, a multivalent gp96 vaccine aiming at cancer prevention could sufficiently generate cross-reacting CTLs against precancerous cells undergoing dysplastic changes with more embryonic phenotypes.

Selecting and confining vaccine targets specific for the tumor, including oncoviral proteins (e.g., HPV, HBV, HCV, EBV, and herpesvirus) ${ }^{2}$, tumor-associated antigens (e.g., MUC1 and HER2) ${ }^{27}$, and tissue-specific self-proteins (e.g., $\alpha$-lactalbumin $)^{3,6}$, represents a major effort in this field. In contrast to the high density but narrowly focused vaccines above, P-gp96 (as a multivalent vaccine with broader antigen distribution but lower individual antigen density) may target multiple antigens likely involved in early dysplastic changes during multiple pre-malignant stages of cancer. In this context, this may represent a strength of this vaccination approach to overcome tumor immunoescape. More studies are required to identify and characterize antigenic peptides associated with P-gp96 and to test the vaccine protection effect against other types of cancer. Also, it is worthwhile to explore if other HSPs (e.g., HSP70) from the placenta exhibit similar immunogenicity against cancer. Considering the possibility that certain embryonic antigens may be viewed as non-self by the immune system and somehow have available $\mathrm{T}$ cell repertoires sufficient to provide tumor protection, this study provides a proof-of-principle for effective prophylactic cancer vaccination for multivalent protection in humans, especially for those with a high risk of neoplastic disease.

\section{Methods}

Human specimens. Normal liver tissues were obtained from the unused portions of five donor livers used for liver transplantation in Beijing You'an Hospital of Capital University of Medical Sciences. Placental samples were collected from five women hospitalized in the Peking University Third Hospital from July, 2011 to December, 2011. Written informed consent was provided by all study participants. The study protocol was approved by the Ethics Committee of Beijing You'an Hospital and the Ethics Committee of the Peking University Third Hospital.

Cell lines. TUBO is a cloned cell line generated from a spontaneous mammary gland tumor from a BALB-neuT mouse and highly expresses HER-2 protein on the cell membrane. TUBO cells were cultured in DMEM (GIBCO) medium containing 10\% fetal calf serum (FBS), $25 \mu \mathrm{g} / \mathrm{ml}$ streptomycin, and $100 \mathrm{IU} / \mathrm{ml}$ penicillin. B16-F10 cells were cultured in RPMI 1640 (GIBCO) medium supplemented with 10\% FBS, $25 \mu \mathrm{g} / \mathrm{ml}$ streptomycin, and $100 \mathrm{IU} / \mathrm{ml}$ penicillin.

Mice and rats. Female BALB/c (6-8 weeks old) and C57BL/6 (6-8 weeks old) mice were purchased from the Animal Research Center of the Medical Department in Peking University (Beijing, China). FVB/N-Tg(MMTVneu)202 Mul/J mice (6-7 weeks old) were kindly provided by Prof. Mingzhao Zhu (Institute of Biophysics, Chinese Academy of Sciences, Beijing China). Female Wistar rats (6-7 weeks old) were obtained from Beijing HFK Bio-technology Co. Ltd. (Beijing, China). All mouse and rat experiments were carried out in strict accordance with the regulation of the Institute of Microbiology, Chinese Academy of Sciences of Research Ethics Committee.

Preparation of gp96. Mouse, rat, and human P-gp96 and mouse L-gp96 were purified by ConA-Sepharose affinity chromatography followed by anion exchange chromatography from mouse, rat, and human placenta or liver tissues, respectively ${ }^{21}$.

Mouse and rat vaccination. In prophylaxis studies, $\mathrm{BALB} / \mathrm{C}$ and $\mathrm{C} 57 \mathrm{BL} / 6$ mice were immunized by subcutaneous injection in the abdominal flank with $20 \mu \mathrm{g}$ P-gp96/ mouse, $20 \mu \mathrm{g} \mathrm{L-gp96/mouse,} \mathrm{or} \mathrm{PBS} \mathrm{alone} \mathrm{at} \mathrm{weeks} \mathrm{1,} \mathrm{2,} \mathrm{and} \mathrm{4.} \mathrm{One} \mathrm{week} \mathrm{after} \mathrm{the}$ third immunization, mice were subcutaneously challenged with $6 \times 10^{5} \mathrm{TUBO}$ cells/ mouse or $5 \times 10^{4} \mathrm{~B} 16-\mathrm{F} 10$ cells/mouse. In therapeutic studies, BALB/c mice were subcutaneously challenged with $6 \times 10^{5}$ TUBO cells/mouse. On the second day, mice were immunized with $20 \mu \mathrm{g} \mathrm{P}$-gp96/mouse, $20 \mu \mathrm{g}$ L-gp96/mouse, or PBS alone three times at 3-day intervals. To evaluate long-lasting protective immunity, $\mathrm{BALB} / \mathrm{c}$ and C57BL/6 mice were immunized by subcutaneous injection in the abdominal flank with $20 \mu \mathrm{g}$ P-gp96/mouse, $20 \mu \mathrm{g} \mathrm{L-gp96/mouse,} \mathrm{or} \mathrm{PBS} \mathrm{alone} \mathrm{at} \mathrm{weeks} \mathrm{1,} \mathrm{2,} \mathrm{and} 4$. Three months after the third immunization, mice were subcutaneously challenged with $6 \times 10^{5} \mathrm{TUBO}$ cells/mouse or $5 \times 10^{4} \mathrm{~B} 16$-F10 cells/mouse. Each group contained at least 10 mice. Tumor size was then measured every other day. Mice were monitored for 50 days for survival.

Female FVB/N-Tg(MMTVneu)202 Mul/J mice were vaccinated by subcutaneous injection in the abdominal flank with $25 \mu \mathrm{g}$ P-gp96/mouse, $25 \mu \mathrm{g} \mathrm{L}$-gp96/mouse, or PBS three times at weeks 1,2 , and 4 . Tumor occurrence and growth was monitored at regular intervals. Female Wistar rats were vaccinated by subcutaneous injection in the abdominal flank with $25 \mu \mathrm{g}$ P-gp96/rat or PBS three times at weeks 1, 2, and 4. One week after the last immunization, mammary carcinogenesis was induced in the rats by providing $20 \mathrm{mg} / 100 \mathrm{~g}$ body weight of DMBA (Sigma) in $1 \mathrm{~mL}$ of corn oil by gavage. The experiment was terminated at 21 weeks after DMBA exposure.

Tumor size was determined by measuring the smallest diameter (a) and the largest diameter (b) by caliper. Tumor volume was calculated using the formula: $\mathrm{V}=\left(\mathrm{a}^{2} \mathrm{~b}\right) / 2$.

DC preparation and immunization. Bone marrow was harvested from femurs and tibias of $\mathrm{C} 57 \mathrm{BL} / 6$ or $\mathrm{BALB} / \mathrm{c}$ mice by flushing the medullary canal with PBS. Bone marrow was filtered through a $100-\mu \mathrm{m}$ nylon cell strainer before erythrocytes were lysed in $0.83 \mathrm{M} \mathrm{NH}_{4}$ CL buffer. Cells were resuspended in RPMI 1640 supplemented with $10 \%$ fetal calf serum, $100 \mathrm{U} / \mathrm{ml}$ penicillin, $100 \mu \mathrm{g} / \mathrm{ml}$ streptomycin, $50 \mathrm{ng} / \mathrm{ml}$ recombinant murine GM-CSF (R\&D Systems) and $20 \mathrm{ng} / \mathrm{ml}$ recombinant murine IL-4 (R\&D Systems) at a concentration of $1 \times 10^{7}$ cells $/ \mathrm{mL}$ and cultured at $37^{\circ} \mathrm{C}$. DC precursors were cultured by the addition of $2 \mathrm{ml}$ of media every two days. On day 7 , DCs were harvested and stimulated with B16-F10 or TUBO tumor cell lysates at a concentration of $50 \mu \mathrm{g}$ protein $/ \mathrm{ml}$ for $24 \mathrm{~h}$. The proportion of DCs in the culture was consistently $90 \%$ as determined by flow cytometric analysis.

$\mathrm{BALB} / \mathrm{c}$ and C57BL/6 mice were immunized by subcutaneous injection in the abdominal flank with $20 \mu \mathrm{g} \mathrm{P}$-gp $96 /$ mouse, $1 \times 10^{6} \mathrm{DCs} /$ mouse, or PBS alone at weeks 1,2 , and 4 . One week after the third immunization, mice were subcutaneously challenged with $6 \times 10^{5}$ TUBO cells/mouse or $5 \times 10^{4} \mathrm{~B} 16-\mathrm{F} 10$ cells/mouse.

Adoptive T cell transfer. Splenocytes were isolated from the spleens of P-gp96immunized, L-gp96-immunized, or unimmunized (PBS) BALB/c mice as described ${ }^{15}$. $\mathrm{CD}^{+} \mathrm{T}$ cells were enriched (>90\%) by magnetic bead separation (Miltenyi, Biotec, Germany) from cultured splenocytes. Purified T cells $\left(2 \times 10^{7}\right)$ were intravenously injected into $\gamma$-irradiated (500 rads) BALB/c mice, followed by subcutaneous challenge with $6 \times 10^{5}$ TUBO cells/mouse. Tumor size was measured at regular intervals, and mice were monitored for 50 days for survival.

Peripheral blood mononuclear cell (PBMC) collection. PBMCs were isolated from freshly heparinized blood of five healthy donors by Ficoll-Hypaque (GE healthcare) density gradient centrifugation.

Enzyme-linked immunosorbent spot (ELISPOT) analysis. Mouse IFN- $\gamma$ and human IFN- $\gamma$ ELISPOT assays were performed according to the protocol supplied by manufacturer (Mabtech, Mariemont, OH). Briefly, isolated mouse splenocytes or human PBMCs (at the indicated number of cells/well pulsed with different stimuli, respectively) were added to the well in triplicate and incubated at $37^{\circ} \mathrm{C}$ for $18-36 \mathrm{~h}$ Spot forming cells (SFCs) were counted and analyzed with an ELISPOT Reader (Biosys, Germany). The indicated control peptides were used as background controls.

T cell cytotoxicity assay. Cytolysis was determined by 5-(6)-carboxy-fluorescein succinimidyl ester (CFSE) and propidium iodide (PI) two fluorochrome staining. Mouse splenocytes or human PBMCs were used as the effector cells. The B16-F10, TUBO, and MCF-7cell lines were used in the assay as target cells. Target cells were pre-labeled with CFSE and mixed with effector cells at various ratios in a final volume of $200 \mu \mathrm{l}$. After 5 -h incubation at $37^{\circ} \mathrm{C}$, dead cells were labeled with PI. Samples were analyzed by fluorescence-activated cell sorting (FACS) using CellQuest software (BD Biosciences).

Real-time PCR analysis. Fresh mouse and rat placenta and liver tissues were obtained from pregnant mice and rats right before RNA extraction. Human placenta and liver tissue samples were fresh frozen in liquid nitrogen before use. Total RNA was extracted from mouse, rat, and human placenta or liver tissues using Trizol reagent (Invitrogen, Carlsbad, CA, USA) according to the manufacturer's instructions. cDNAs were generated from $500 \mathrm{ng}$ of total RNA using Superscript RT reagent kit (Takara). Real-time fluorescence quantitative PCR was performed using SYBR Green Premix Reagent (Takara Bio Inc., Shiga, Japan). The PCR conditions were $95^{\circ} \mathrm{C}$ for 30 seconds, followed by 40 cycles of $95^{\circ} \mathrm{C}$ for 15 seconds and $60^{\circ} \mathrm{C}$ for 45 seconds. GAPDH and $\beta$-actin were used as internal standards for quantification. PCR primers specific for HER2, MUC1, MMP2, FGF2, c-Myc and VEGF-a were designed by online tool (http://www.idtdna.com/scitools/Applications/RealTimePCR/) and synthetized by Invitrogen. All primer sequences are listed in Supplementary Table S2. Data for estimation of gene expression between mouse, rat, and human placenta tissues and liver tissues were analyzed using the modified ${ }^{\Delta \Lambda} \mathrm{C}_{\mathrm{t}}$ method to generate a quantity for gene expression in placenta tissue relative to gene expression in liver tissue $e^{28,29}$.

Immunocytochemistry. Rat mammary tumors, fixed in $4 \%$ buffered formalin, were embedded in paraffin using a conventional automated system. Paraffin-embedded tissues (5- $\mu \mathrm{m}$ sections) were stained with hemotoxylin-eosin.

Statistical analysis. The values are expressed as means \pm s.d. Differences of the mean tumor volume, SFC, percentage of killing, and relative mRNA expression levels among PBS-, P-gp96- and L-gp96-treated mice were compared with the Student's 
t-test and analysis of variance (ANOVA) and post-hoc comparison using Tukey's test. Differences between Kaplan-Meier curves were compared with the log-rank test.

1. O'Callaghan, T. Introduction: The prevention agenda. Nature 471, S2-4 (2011).

2. Liu, M. A. Cancer vaccines. Philos Trans R Soc Lond B Biol Sci 366, 2823-2826 (2011).

3. Tuohy, V. K. \& Jaini, R. Prophylactic cancer vaccination by targeting functional non-self. Ann Med 43, 356-365 (2011).

4. Pere, H. et al. A CCR4 antagonist combined with vaccines induces antigenspecific CD8(+) T cells and tumor immunity against self antigens. Blood 118, 4853-4862 (2011)

5. Nanni, P. et al. Antimetastatic activity of a preventive cancer vaccine. Cancer Res 67, 11037-11044 (2007).

6. Jaini, R. et al. An autoimmune-mediated strategy for prophylactic breast cancer vaccination. Nat Med 16, 799-803 (2010)

7. Brewer, B. G., Mitchell, R. A., Harandi, A. \& Eaton, J. W. Embryonic vaccines against cancer: An early history. Exp Mol Pathol 86, 192-197 (2009).

8. Dong, W. et al. Administration of embryonic stem cells generates effective antitumor immunity in mice with minor and heavy tumor load. Cancer Immunol Immun 59, 1697-1705 (2010).

9. Yaddanapudi, K. et al. Vaccination with Embryonic Stem Cells Protects against Lung Cancer: Is a Broad-Spectrum Prophylactic Vaccine against Cancer Possible? PloS one 7, e42289 (2012).

10. Garcia-Hernandez Mde, L., Gray, A., Hubby, B., Klinger, O. J. \& Kast, W. M. Prostate stem cell antigen vaccination induces a long-term protective immune response against prostate cancer in the absence of autoimmunity. Cancer Res $\mathbf{6 8}$, 861-869 (2008)

11. Sood, R., Zehnder, J. L., Druzin, M. L. \& Brown, P. O. Gene expression patterns in human placenta. P Natl Acad Sci USA 103, 5478-5483 (2006).

12. Robert, J., Ramanayake, T., Maniero, G. D., Morales, H. \& Chida, A. S. Phylogenetic conservation of glycoprotein 96 ability to interact with CD91 and facilitate antigen cross-presentation. J Immunol 180, 3176-3182 (2008).

13. Kropp, L. E., Garg, M. \& Binder, R. J. Ovalbumin-derived precursor peptides are transferred sequentially from gp96 and calreticulin to MHC class I in the endoplasmic reticulum. J Immunol 184, 5619-5627 (2010).

14. Meng, S. D., Gao, T., Gao, G. F. \& Tien, P. HBV-specific peptide associated with heat-shock protein gp96. Lancet 357, 528-529 (2001).

15. Liu, Z. et al. Treg suppress CTL responses upon immunization with HSP gp96. Eur Immunol 39, 3110-3120 (2009).

16. Pawaria, S. \& Binder, R. J. CD91-dependent programming of T-helper cell responses following heat shock protein immunization. Nat Commun 2, 521 (2011).

17. Yang, Y. et al. Heat shock protein gp96 is a master chaperone for toll-like receptors and is important in the innate function of macrophages. Immunity 26, 215-226 (2007).

18. Warger, T. et al. Interaction of TLR2 and TLR4 ligands with the N-terminal domain of Gp96 amplifies innate and adaptive immune responses. J Biol Chem 281, 22545-22553 (2006).
19. Binder, R. J. \& Srivastava, P. K. Essential role of CD91 in re-presentation of gp96chaperoned peptides. P Natl Acad Sci USA 101, 6128-6133 (2004).

20. Randazzo, M., Terness, P., Opelz, G. \& Kleist, C. Active-specific immunotherapy of human cancers with the heat shock protein Gp96-revisited. Int. J. Cancer 130, 2219-2231 (2012).

21. Meng, S. D., Song, J., Rao, Z. H., Tien, P. \& Gao, G. F. Three-step purification of gp96 from human liver tumor tissues suitable for isolation of gp96-bound peptides. J Immunol Methods 264, 29-35 (2002).

22. Yan, X. L. et al. Regulatory T-cell depletion synergizes with gp96-mediated cellular responses and antitumor activity. Cancer Immunol Immun 60, 1763-1774 (2011).

23. Manjili, M. H. et al. HSP110-HER2/neu chaperone complex vaccine induces protective immunity against spontaneous mammary tumors in HER-2/neu transgenic mice. J Immunol 171, 4054-4061 (2003).

24. Jacob, J. et al. Activity of DNA vaccines encoding self or heterologous Her-2/neu in Her-2 or neu transgenic mice. Cell Immunol 240, 96-106 (2006).

25. Gong, J. L. et al. A heat shock protein 70-based vaccine with enhanced immunogenicity for clinical use. J Immunol 184, 488-496 (2010).

26. Hobohm, U. Toward general prophylactic cancer vaccination. Bioessays 31 1071-1079 (2009).

27. Eisenstein, M. Vaccines: Know your enemy. Nature 471, S8-S9 (2011).

28. Hao, J. et al. Inhibition of alpha interferon (IFN-alpha)-induced microRNA-122 negatively affects the anti-hepatitis B virus efficiency of IFN-alpha. J Virol 87, 137-147 (2013)

29. Livak, K. J. \& Schmittgen, T. D. Analysis of relative gene expression data using real-time quantitative PCR and the 2(T)(-Delta Delta C) method. Methods 25, 402-408 (2001)

\section{Acknowledgements}

This work was supported by grants from the National Natural Science Foundation of China $(31230026,30970146,91029724,81021003$, and 81102018).

\section{Author contributions}

S.M. designed the study. B.Z., Y.W. carried out experiments and analyzed data with the help from B.W., E.W., H.F., M.G., L.C., C.L. and Y.J. S.L. and W.Z. provided data or study samples. S.M. and B.Z. prepared the manuscript. All authors reviewed the manuscript.

\section{Additional information}

Supplementary information accompanies this paper at http://www.nature.com/ scientificreports

Competing financial interests: The authors declare no competing financial interests.

License: This work is licensed under a Creative Commons

Attribution-NonCommercial-NoDerivs 3.0 Unported License. To view a copy of this license, visit http://creativecommons.org/licenses/by-nc-nd/3.0/

How to cite this article: Zhao, B. et al. Placenta-derived gp96 as a multivalent prophylactic cancer vaccine. Sci. Rep. 3, 1947; DOI:10.1038/srep01947 (2013). 